\title{
„Schülerjob“ revisited: Zur Passung von Lehr- und Lernhabitus im Unterricht
}

\author{
Matthias Martens • Barbara Asbrand
}

Eingegangen: 1. Oktober 2020 / Überarbeitet: 18. April 2021 / Angenommen: 20. April 2021 / Online publiziert: 17. Mai 2021

(C) Der/die Autor(en) 2021

Zusammenfassung Der „Schülerjob“ gilt seit der vielzitierten Publikation von Georg Breidenstein als eine der zentralen theoretischen Kategorien zur Beschreibung der Bewältigung von unterrichtlichen Anforderungen durch Schüler/innen. Der Beitrag verknüpft das in der ethnografischen Forschung erarbeitete Konzept mit der kultur- und wissenssoziologisch fundierten dokumentarischen Unterrichtsforschung. Dabei wird die grundlegende Frage weiterverfolgt, wie Schüler/innen mit den unterrichtlichen Anforderungen umgehen. In verschiedenen Studien haben wir eine an das Konzept des Schülerjobs angelehnte „Orientierung an der Aufgabenerledigung“ als Basistypik von Unterricht herausgearbeitet. In den auf unterschiedliche Forschungsfragen fokussierten Studien haben wir die empirische Perspektive geweitet und das Verhältnis der mit Bezug auf den Schülerjob beschreibbaren Lernhabitus der Schüler/innen einerseits und die im Unterricht rekonstruierbaren Lehrhabitus der Lehrpersonen andererseits in den Blick genommen. Auf diese Weise ist eine Ausdifferenzierung des Schülerjobs gelungen: Der Modus der Aufgabenerledigung variiert in Abhängigkeit von den etablierten Passungsverhältnissen von Lehr- und Lernhabitus. Der Aufsatz leistet eine Systematisierung von Ergebnissen, die in unterschiedlichen Studien vorliegen, in Form einer Typik und versteht sich somit als Beitrag zur Theoriebildung zum Schülerjob.

Prof. Dr. Matthias Martens ( $\square)$

Empirische Schulforschung mit dem Schwerpunkt Unterrichtsentwicklung, Department für Erziehungs- und Sozialwissenschaften, Humanwissenschaftliche Fakultät, Universität zu Köln, Aachener Str. 201, 50931 Köln, Deutschland

E-Mail: m.martens@uni-koeln.de

Prof. Dr. Barbara Asbrand

Erziehungswissenschaft mit dem Schwerpunkt Allgemeine Didaktik und Schulentwicklung, Fachbereich Erziehungswissenschaften, Goethe-Universität Frankfurt am Main, Theodor W. Adorno Platz 6, 60629 Frankfurt am Main, Deutschland 
Schlüsselwörter Schülerjob · Aufgabenerledigung · Dokumentarische Unterrichtsforschung · Lehr- und Lernhabitus · Typenbildung

\title{
"Student's job" revisited: complementary relations of teaching and learning habitus in the classroom
}

\begin{abstract}
Since the much-cited publication by Georg Breidenstein, "Schülerjob" has been considered one of the central theoretical categories for describing how students cope with demands and expectations of teaching. This contribution links the concept developed in ethnographic research with documentary research on classroom interaction, which is based on theories of cultural sociology and sociology of knowledge. The fundamental question of how students deal with the demands of teaching is pursued further. In various studies, we have developed an "orientation towards the completion of tasks" based on the concept of the student's job as a basic type of teaching. In the studies, which focused on different research questions, we broadened the empirical perspective and looked at the relationship between the learning habitus of the students that can be described with reference to the student job on the one hand and the teaching habitus of the teachers that can be reconstructed in class on the other. In this way, a differentiation of the student's job has been achieved: The mode of task completion varies depending on the established relation between teaching and learning habitus. The paper systematizes the results of different studies in the form of a typology and is thus a contribution to the theory of the student's job.
\end{abstract}

Keywords Student's job/doing student · Completion of tasks · Documentary classroom research $\cdot$ Teaching and learning habitus $\cdot$ Type-formation

\section{Einleitung}

Der „Schülerjob“ ist seit der Publikation von Georg Breidenstein (2006) eine zentrale theoretische Kategorie, um die Bewältigung unterrichtlicher Anforderungen durch Schüler/innen $\mathrm{zu}$ beschreiben. Hintergrund ist eine ethnografische Forschungstradition, die eine Hierarchisierung der Lehrpersonen- gegenüber der Schüler/innenperspektive als Wahrnehmungsroutine mit erkenntnishemmender Wirkung kritisiert hatte (z. B. Jackson 1975; Zinnecker 1978; vgl. Breidenstein 2008). Während die ethnografische Schüler/innenforschung der 1980er-Jahre von einer Betonung des subversiven oder widerständigen Charakters der Schülerwelten im Sinne einer Gegenkultur geprägt war (z. B. Zinnecker 1978; Heinze 1980), verfolgt Breidenstein mit seiner Studie zum Schülerjob ,,eine symmetrischere Betrachtungsweise [...], die es erlaubt, die Kooperation der Beteiligten bei der Aufrechterhaltung einer bestimmten Ordnung in den Blick zu nehmen." (Breidenstein 2006, S. 15). Für ihn ist daher die Fragestellung leitend, wie die „Praktiken von Schülern und Lehrpersonen aufeinander bezogen und ineinander verschränkt [sind], so dass sie gemeinsam den ,Unterricht" ermöglichen“ (ebd.). Dieser Fokus ermöglicht 
es, Spannungsverhältnisse zwischen der Distanzierung gegenüber den schulischen Anforderungen und der Übernahme schulischer Handlungsmuster aufzudecken.

Hintergrund für eine Ausdifferenzierung des Schülerjobs, die wir in diesem Beitrag vorschlagen, bilden mehrere erziehungswissenschaftliche und fachdidaktische Studien der dokumentarischen Unterrichtsforschung (Asbrand und Martens 2018), die an unserem Arbeitsbereich entstanden sind. Diese Forschungsprojekte beschäftigten sich zwar mit unterschiedlichen gegenstandsbezogenen Fragestellungen, dennoch wurde studienübergreifend eine an das Konzept des Schülerjobs anschlussfähige „Orientierung an der Aufgabenerledigung“ als Basistypik für die Beteiligung von Schüler/innen am Unterricht herausgearbeitet (vgl. z. B. Spieß 2014; KaterWettstädt 2015; Petersen 2015; Martens 2015, 2018, 2020; Gresch 2020; Gresch und Martens 2019; Schreyer et al. eingereicht). In diesem Aufsatz schließen wir uns Breidensteins Forschungsfrage nach dem Umgang der Schüler/innen mit den unterrichtlichen Anforderungen an, fragen aber vertiefend nach den situativen und strukturellen Rahmenbedingungen der Ausbildung des Schülerjobs und können ihn dadurch in verschiedenen typischen Ausprägungen beschreiben. Verknüpft werden dabei die Befunde der ethnografischen und der dokumentarisch-wissenssoziologischen Unterrichtsforschung auf der Ebene der Rekonstruktionsergebnisse im Sinne einer empirisch basierten, gegenstandsbezogenen Theoriebildung.

In diesem Aufsatz werden wir im Anschluss an eine Darstellung des Forschungsstands zur Bewältigung unterrichtlicher Anforderungen im „Job-Modus“ (Kap. 2) die Basistypik „Orientierung an Aufgabenerledigung“ und die Passungsverhältnisse von Lehr- und Lernhabitus theoretisch und anhand eines empirischen Beispiels vorstellen (Abschn. 3.1). Anschließend werden wir die in den unterschiedlichen Studien rekonstruierten Ausprägungen der Basistypik darstellen (Abschn. 3.2). Diese relationale Typenbildung (vgl. Nohl 2013a) leistet eine studienübergreifende Systematisierung und Abstrahierung von Einzelergebnissen, ordnet diese theoretisch ein und versteht sich somit als Beitrag zur Theoriebildung zum Schülerjob.

\section{Bewältigung unterrichtlicher Anforderungen im Job-Modus}

Breidenstein fasst den Umgang der Schüler/innen mit unterrichtlichen Anforderungen metaphorisch als „Schülerjob“. Diese Metapher nimmt auf, dass die ArbeitsTerminologie im Unterricht allgegenwärtig ist (vgl. Breidenstein und Menzel 2014) und fasst die durch Routine und Distanz gekennzeichneten ,fraglos durchgeführten, unspektakulären Verrichtungen, Aufgaben und Abläufe, die den alltäglichen Unterricht konstituieren“" (Breidenstein 2006, S. 16).

Schon ältere Studien haben das Verhältnis von Routine und Distanz in Bezug auf die unterrichtlichen Anforderungen untersucht. Jackson (1973, 1975) skizziert die „Welt des Schülers“ als komplementär zu typischen Verhaltensweisen von Lehrpersonen. Aus der strukturellen Knappheit der „Ressource Lehrperson“ (vgl. auch Breidenstein et al. 2012) ergeben sich für die Schüler/innen fast zwangsläufig Erfahrungen des „Leerlaufs“ und des Wartens, Erfahrungen von Unterbrechungen der Arbeitsprozesse sowie Erfahrungen des Versagens und der Zurückweisung von eigenen (Lern-)Bedürfnissen. Die Schüler/innen reagieren hierauf mit spezifischen 
„Anpassungsprozessen“ (Jackson 1975, S. 27) in Form von Geduld, Resignation und „Maskierung“ (ebd., S. 28). Die Schüler/innen verwandeln sich in „Schulstuben-Profis“ (Jackson 1973, S. 21), die es perfektioniert haben, durch ,geheimnisvolles Schweigen und lässiges Herumhängen Enthusiasmus wie Desinteresse am Bildungsgeschehen wirksam [zu] kaschieren." (ebd.). Gegenüber der Lehrperson ist die Maskierung besonders notwendig, weil der Unterricht ,ein Bewertungsfeld [ist], in dem der Schüler nicht nur lernen muß, sich Befehlen zu fügen, sondern so zu fügen, dass eine positive Bewertung daraus folgt. So entsteht die Gepflogenheit, dem Lehrer in Klassenarbeiten und Prüfungen das Seine zu geben - auch wenn die Aufgaben sinnlos und die Fragen blödsinnig erscheinen“ (ebd.).

Heinze (1980) hebt hervor, dass im Unterricht regelmäßig ,der erfahrungsgemäße Charakter von Lernen [...] verletzt [wird]. Daraus resultieren [...] Motivierungsschwäche, latente Widerstände, Fremdbestimmung und Desinteresse, äußere Anpassung an Leistungsanforderungen ohne persönliche Beziehung zu den Lerngegenständen usw." (ebd., S. 17f.). Mit Bezug auf Goffman (1971) macht Heinze deutlich, dass die Erwartung der Lehrperson an ein angemessenes unterrichts- bzw. anforderungsbezogenes Engagement durch „Schülertaktiken“ unterlaufen wird. So entwickeln Schüler/innen ein hohes Maß an Routine darin, ein ,abgeschirmtes Engagement“ zu etablieren, nämlich das gekonnte Verbergen von inadäquatem Engagement und die gleichzeitige Zur-Schaustellung von angemessenem Verhalten (Heinze 1980, S. 86).

Maskierung und abgeschirmtes Engagement sind Praktiken von Schüler/innen, die sich auch in Breidensteins „Schülerjob“ wiederfinden. Als dessen Hauptcharakteristikum benennt er, dass ,,[m]an tut, was zu tun ist, ohne damit (vollständig) identifiziert zu sein. Man kommt seinen Verpflichtungen nach, ohne grundsätzlich nach Sinn und Legitimität zu fragen, und das alltägliche Tun ist wesentlich durch Routine und Pragmatismus geprägt“ (Breidenstein 2006, S. 11). Über alle beobachteten Unterrichtsformate hinweg beschreibt Breidenstein (2006) den Kern des Schülerjobs als „Produktionsorientierung“ (ebd., S. 214). Kennzeichnend ist die möglichst effektive und rationale Erstellung des Arbeitsergebnisses, die sich (gegenüber der durch die Lehrperson möglicherweise intendierten fachlichen Sinnbildung) zu verselbstständigen und einen Wert an sich darzustellen scheint.

Die hier vorgestellten empirischen Befunde lassen sich mit Luhmann (2002, S. 77f.) theoretisch durch Trivialisierungen erklären, die das Erziehungssystem durch seinen Anspruch, Veränderungen bei psychischen Systemen hervorzurufen, zwangsläufig erzeugt. Zu Trivialisierungen kommt es einerseits, weil das zu lernende Wissen eindeutig definiert ist und Schülerantworten als falsch oder richtig eingeordnet werden können, andererseits weil nicht nur Wissen, sondern auch eine Wertschätzung des schulischen Wissens erwartet wird (ebd., S. 79; vgl. auch Bourdieu und Passeron 1971). Die Struktur des Unterrichts als Interaktion unter Anwesenden führt dazu, dass die Schüler/innen erwarten, dass Akzeptanz und Wertschätzung der Sache von ihnen erwartet wird und sie stellen sich auf die Erfüllung dieser Erwartungen ein. Schüler/innen lernen also, mit den an sie gerichteten, tendenziell trivialisierenden Erwartungen umzugehen, indem sie ihnen gerecht werden, ohne sich mit ihnen zu identifizieren (Luhmann 2002, S. 80). 


\section{Aufgabenerledigung in Passungsverhältnissen von Lehr- und Lernhabitus}

\subsection{Theoretischer Hintergrund und Beispielinterpretation}

Mit Bezug auf die Schülerjob-Metapher schließen wir uns einer Sichtweise an, die Unterricht als eine heteronome Erwartungs- und Anforderungsstruktur versteht, die auf Schüler/innenseite spezifische Bewältigungsmuster hervorbringt. Übertragen auf Vorstellungen eines Angebots-Nutzugs-Modells des Unterrichts (Helmke 2017) stehen also jene Routinen der Schüler/innen im Fokus, die auf die Wahrnehmung und Interpretation des „Angebots“ ausgerichtet sind und die die „Nutzung“ dieses Angebots strukturieren. In Erweiterung zur Studie von Breidenstein (2006) nehmen wir zusätzlich auch die „Angebotsseite“ genauer in den Blick und fragen nach den Routinen und habituellen Mustern, nach denen Lehrpersonen den Unterricht als Erwartungs- und Anforderungsstruktur (mit)hervorbringen. Vor diesem Hintergrund rekonstruieren wir im Rahmen der dokumentarischen Unterrichtsforschung Lehr- und Lernhabitus als Dimensionen der Lehrer/innen- und Schüler/innen-Habitus sowie deren Hervorbringung und Verwobenheit in der Interaktion. Der Fokus ist - anders als in Forschung zum Lehrpersonen- und Schülerhabitus (z. B. Helsper et al. 2014; Kramer und Pallesen 2019) - auf das soziale Geschehen im Unterricht gerichtet. Die Lehr- und Lernhabitus sind in der Unterrichtsinteraktion nach unseren bisherigen empirischen Analysen in der Regel - nicht zwangsläufig - in Passungsverhältnissen aufeinander bezogen, in denen sich eine Komplementarität der sozialen Rollen und der konjunktiven Erfahrungsräume des Unterrichts zeigt (Martens und Asbrand 2017). ${ }^{1}$

Unter Lehrhabitus verstehen wir - in Anlehnung an den Habitusbegriff Bourdieus (1982) - die auf das Unterrichten bezogenen verinnerlichten Wahrnehmungs-, Denk- und Handlungsmuster, die die Unterrichtspraxis lehrpersonenseitig strukturieren. Rekonstruiert wird das in der Unterrichtsinteraktion rekonstruierbare (pädagogisch-)praktische Wissen und Können der Lehrperson (vgl. Helsper 2001, 2018). Der Lehrhabitus dokumentiert sich allerdings nicht nur in verbalen und korporierten Äußerungen der Lehrperson, sondern lässt sich im Rahmen einer videobasierten dokumentarischen Unterrichtsforschung (vgl. zum methodischen Vorgehen ausführlich Asbrand und Martens 2018) z. B. auch in der Analyse von Artefakten, Lehrmaterialien und Aufgaben, die die Lehrperson für den Unterricht selektiert, rekonstruieren. Aus diesem Grund können gleichzeitig unterschiedliche Lehrhabitus im Unterricht präsent sein, die kongruent oder inkongruent zueinander stehen können. Die Lehrhabitus können sich darüber hinaus sowohl konsistent als auch inkonsistent zu den Orientierungsschemata verhalten, die den Unterricht auf der Ebene der (fach-)didaktischen und pädagogischen Normen und Programmatiken bestimmen.

\footnotetext{
1 Im Vergleich zu Studien von z. B. Kramer (2014, 2015), Bremer und Lange-Vester (2014) oder Gibson und Helsper (2018) bewegen wir uns mit unseren empirischen Studien zu unterrichtlichen Passungsverhältnissen auf der Mikroebene der Herstellung kultureller Passungen zwischen primären (familialen, herkunftsspezifischen) und sekundären (schulbezogenen) Habitus.
} 
Mit Lernhabitus sind die auf das fachliche Lernen bzw. die Lernanforderungen bezogenen Wahrnehmungs-, Denk- und Handlungsmuster bezeichnet, die den Unterricht seitens der Schüler/innen strukturieren. Auch hier sind in der Regel mehrere unterschiedliche Ausprägungen von Lernhabitus gleichzeitig im Unterricht präsent und überlagern sich zudem mit anderen Dimensionen des Schüler/innenhabitus (z. B. herkunftsbezogenen, peerkulturellen, oder schulformbezogenen Orientierungen, vgl. Thiersch 2014; von Rosenberg 2014; Helsper et al. 2020).

Als wesentliche Ausprägung des Lernhabitus konnten wir studienübergreifend (Petersen und Asbrand 2013; Spieß 2014; Wettstädt und Asbrand 2014; Kater-Wettstädt 2015; Petersen 2015; Martens 2015, 2018, 2020; Hackbarth 2017; Gresch 2020; Gresch und Martens 2019; Kreft 2020; Schreyer et al. eingereicht; Asbrand et al. 2018; Schuster in Vorbereitung) eine „Orientierung an der Aufgabenerledigung“ rekonstruieren, die darin besteht, das unterrichtliche „Angebot“ vor allem in seinem heteronomen Auf- und Anforderungsgehalt wahrzunehmen und dieses mit mehr oder weniger Distanz und Pragmatismus erledigend $\mathrm{zu}$ bearbeiten. Es wird dabei deutlich, dass im Verhältnis zu den im Angebot gesetzten (fachlichen) Relevanzen eigenlogische Relevanzverschiebungen beobachtbar sind (z. B. in Hinsicht auf ein erfolgreiches Abschneiden in der Klassenarbeit). Methodisch hat die „Orientierung an der Aufgabenerledigung" für den vorliegenden Beitrag die Funktion eines tertium comparationis: Das in allen Fällen gefundene Gemeinsame bildet einen Vergleichshorizont, vor dessen Hintergrund Kontraste in einzelnen Fällen deutlich werden (vgl. Nohl 2013b; Bohnsack 2014). Auf diese Weise ist es möglich, eine Typik der Aufgabenerledigung auszuarbeiten und dieses Phänomen der schüler/ innenseitigen Unterrichtsteilnahme differenziert zu beschreiben.

Ein zweites für diesen Beitrag relevantes studienübergreifendes empirisches Ergebnis ist, dass sich Lehr- und Lernhabitus in der Regel zueinander in Passung befinden (Martens und Asbrand 2017). Mit dem Begriff des Passungsverhältnisses meinen wir nicht pädagogisch-normativ gewünschte Entsprechungen, sondern wir bezeichnen mit dem Begriff in einem deskriptiven Sinn den beobachtbaren Zusammenhang von differenten, sich aber ergänzenden Lehr- und Lernhabitus, die sich in asymmetrischen und komplementären Unterrichtsinteraktionen rekonstruieren lassen. Im Fokus steht also die Frage, welche Verhältnisse die gewählten, empirisch beobachtbaren Anschlüsse in der Unterrichtsinteraktion konstituieren. Wir haben Lehren und Lernen empirisch und theoretisch als voneinander unterscheidbare jeweils situative und kontingente sowie gleichermaßen habitualisierte Praxen rekonstruiert. Die Unterrichtsinteraktion erscheint uns folglich als ein für Lehrpersonen und Schüler/ innen unterschiedlicher und je spezifischer konjunktiver Erfahrungsraum (Mannheim 1980). Wichtig ist hervorzuheben, dass die in unseren Studien rekonstruierten Passungsverhältnisse nicht für den gesamten Unterricht gelten, sondern immer auf bestimmte Interaktionssysteme bzw. in der Zeitdimension situativ begrenzt bleiben. Empirisch hat sich gezeigt, dass im Unterricht synchron unterschiedliche Passungsverhältnisse koexistieren, wenn also etwa ein und derselbe Arbeitsauftrag von verschiedenen Schüler/innen(-gruppen) entsprechend ihrer Lernhabitus unterschiedlich interpretiert wird und damit unterschiedliche Typen von Aufgabenerledigung beobachtbar werden. Insofern kann zwar festgehalten werden, dass sich eine bestimmte Ausprägung des Lehrhabitus mit einer bestimmten Ausprägung des Lernhabitus in 


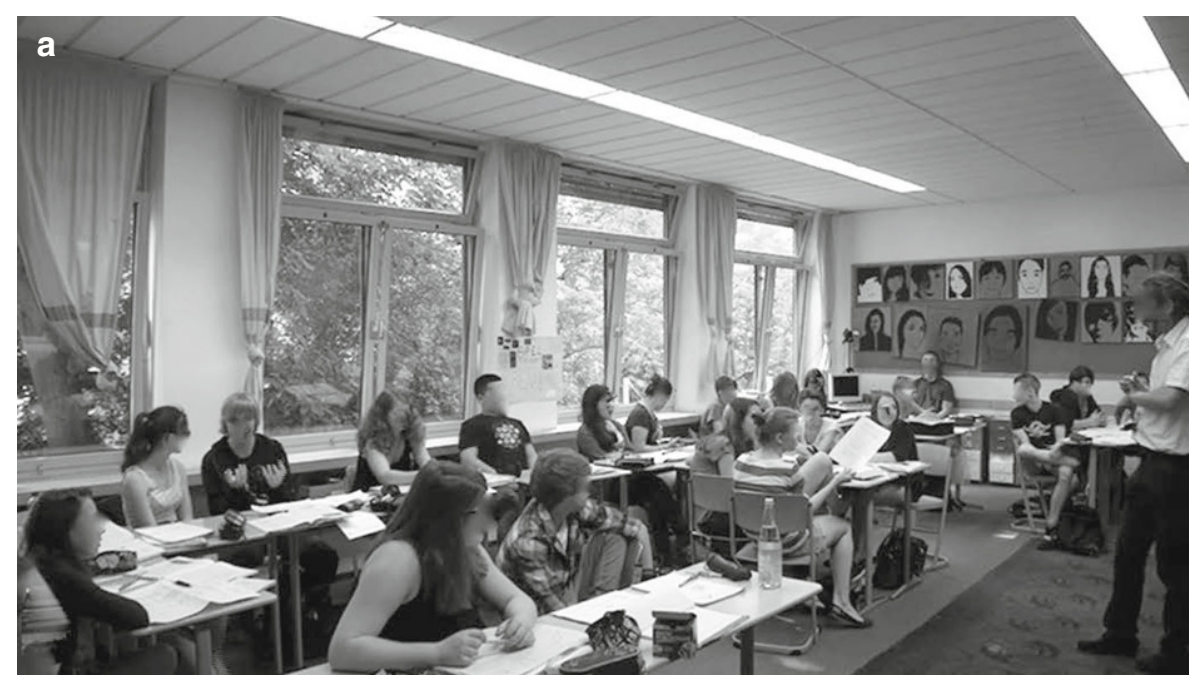

b

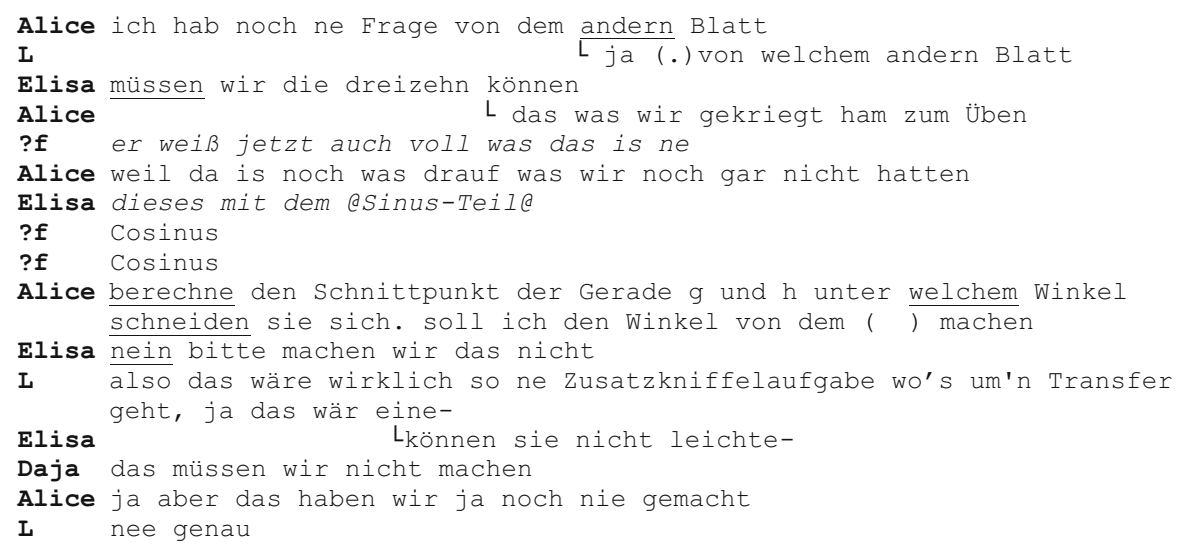

Abb. 1 Aufgabe auf dem Extrazettel (01:18:05)

Passung befindet, aufgrund der kontingenten Sozialdynamiken des Unterrichts aber eher im Verhältnis einer Bedingung der Möglichkeit und nicht in einem determinierenden Zusammenhang. Die Komplexität des Unterrichts zeigt sich darüber hinaus auch darin, dass die Lehrer-Schüler-Interaktionen auch durch tiefere Inkongruenzen der Habitus gekennzeichnet sein können, infolge derer die Interaktion lediglich machtförmig aufrechterhalten wird. Möglich ist auch, dass die Interaktion auf der Ebene der sozialen Ordnung des Unterrichts komplementär verläuft und sich Lehrund Lernhabitus auf dieser Ebene unterscheiden, Lehrpersonen und Schüler/innen aber in einer anderen Dimension, z.B. hinsichtlich des fachlichen Gegenstands, einen Orientierungsrahmen teilen. 
Die Rekonstruktion der Passung von Lehr- und Lernhabitus werden wir im Folgenden an einem empirischen Beispiel illustrieren. ${ }^{2}$ Das Beispiel eignet sich u.E. auch, um aufzuzeigen, wie sich innerhalb einer Lerngruppe schüler/innenseitig unterschiedliche Lernhabitus rekonstruieren lassen. Deutlich wird auch die Passung mehrerer Lernhabitus an einen Lehrhabitus und die mehrdimensionale Überlagerung von Habitusdimensionen. Die Interpretation der Sequenz „Vorbereitung auf die Klassenarbeit" fokussiert die Interaktion der Mädchen an der Tischgruppe in der Klassenraummitte mit der Lehrperson (Abb. 1). Die Schülerinnen sprechen zunächst leise untereinander über eine Aufgabe, die sie als zu schwierig empfinden. Dann adressiert Alice eine Frage an die Lehrperson, woraufhin dieser sich der Tischgruppe zuwendet und die Frage öffentlich diskutiert wird. ${ }^{3}$

In der Interaktion wird auf die Leistungsanforderungen der bevorstehenden Klassenarbeit fokussiert (im Vergleich zum gleichzeitig klassenöffentlich verhandelten Unterrichtsthema eine eigenlogische Relevanzsetzung der hier fokussierten Schülerinnen). Die Schülerinnen erwarten, dass die Lehrperson die notwendigen Rechenverfahren so im Unterricht behandelt, dass sie die Aufgaben lösen können. In der Frage nach den Leistungsanforderungen dokumentiert sich die geteilte Orientierung der Schüler/innen an Aufgabenerledigung, insofern hier der heteronome Anforderungscharakter (die bevorstehende Klassenarbeit) und dessen Entsprechung im Vordergrund steht. In der Sequenz zeigt sich aber auch, wie zusätzlich andere, von den Schülerinnen nicht geteilte Orientierungsdimensionen wirksam sind: Elisa und Daja bezweifeln, dass die fragliche Aufgabe für sie überhaupt eine Anforderung darstellt. Dies zeigt sich in der direkten Frage, ob das Können der Aufgabe ein „Muss“ ist, und in der Fokussierung auf den formalen Charakter der Aufgabe, der eine Distanz zum fachlichen Inhalt impliziert. Dagegen thematisiert Alice die Aufgabe auch in ihrem fachlichen Gehalt und bringt zum Ausdruck, dass ihr Nicht-Können darauf beruht, dass mathematische Konzepte noch nicht im Unterricht behandelt wurden. Damit einher geht der Wunsch, die Aufgabe zu verstehen und die Anforderung des Unterrichts erfüllen zu können. Die unterschiedlichen Leistungsorientierungen der Mädchen können mit dem Gegensatz von ,etwas nicht können müssen“ vs. „etwas nicht können können“ auf den Begriff gebracht werden.

In der verbalen Reaktion auf die Äußerungen der Schülerinnen charakterisiert der Lehrer die fragliche Aufgabe als eine ,Zusatzkniffelaufgabe wo's um'n Transfer geht“. Die Leistungsanforderung der Lehrperson, wonach von leistungsstarken Schüler/innen eigenständige Transferleistungen erwartet werden, verhält sich komplementär zu beiden Leistungsorientierungen der Schülerinnen: Der aufgespannte

\footnotetext{
2 Die hier vorgestellte Interpretation einer Szene aus dem Mathematikunterricht einer achten Klasse in einer Integrierten Gesamtschule ist bereits publiziert. Die ausführliche Interpretation kann in Martens (2015) bzw. in Asbrand und Martens (2018) nachgelesen werden. Methodisch folgt die Interpretation unserem Vorschlag für die Analyse von Unterrichtsvideografien mit der Dokumentarischen Methode (vgl. Asbrand und Martens 2018), die methodische Vorgehensweise kann in diesem Beitrag, der auf die Typisierung und Theoretisierung der rekonstruierten Befunde abzielt, aus Platzgründen nicht ausführlich dargestellt werden und ist an anderer Stelle nachzulesen (ebd.).

3 Im Transkipt kursiv gesetzte Passagen zeigen an, dass das hier Transkribierte bewusst leise gesprochen und nur innerhalb der Tischgruppe verständlich ist. Die Namen der Schüler/innen und der Lehrperson sind anonymisiert.
} 
Gegenhorizont von „Zusatzaufgabe“ und „Pflichtaufgabe“ korrespondiert einerseits mit der Haltung des „Nicht-Können-Müssens“: Zusatzaufgaben sind kein „Muss“. Der Gegenhorizont von Regel- und Transferanforderungen korrespondiert andererseits mit der Leistungsaspiration von Alice, Bea und Clara. Die für die Aufgabe erforderliche Transferleistung ist für den Lehrer gerade dadurch gekennzeichnet, dass die entsprechende Aufgabe also bisher nicht Unterrichtsgegenstand gewesen ist. Insofern befindet sich das „Nicht-Können-Können“ aber „Können-Wollen“ von Alice in Passung zu der vom Lehrer gesetzten Leistungsanforderung für leistungsstarke Schüler/innen.

In der Folge bricht die Lehrperson die Interaktion mit der Mädchengruppe ab und wendet sich mit einer Generalisierung des angesprochenen Problems an die gesamte Klasse, indem er allgemein auf Anforderungsbereiche in Leistungsüberprüfungen rekurriert. Darin zeigt sich, dass Leistungsdifferenzierungen und differente Leistungspositionierungen für die Lehrperson legitim sind, die Klassenarbeit wird eine Hierarchisierung von leistungsstarken und leistungsschwächeren Schüler/innen hervorbringen. Im Blick auf die bevorstehende Klassenarbeit formuliert die Lehrperson klare Anforderungen. Die Orientierung der Mädchen an Aufgabenerledigung, die in dem Wunsch zum Ausdruck kommt, sich bestmöglich auf die Klassenarbeit vorzubereiten, befindet sich dazu in Passung. Das Verhältnis von Lehr- und Lernhabitus ist dabei von der Bereitschaft der Schülerinnen bestimmt, an der Unterrichtsinszenierung der Lehrperson mitzuwirken und den darin implizierten Anforderungen zu entsprechen.

\subsection{Typologie der Aufgabenerledigung}

Im studienübergreifenden, empirischen Fallvergleich mit dem Fokus auf Unterschiede im Gemeinsamen (zum empirischen Vorgehen bei der Typenbildung vgl. Bohnsack 2013; Nohl 2013a, b) ist es gelungen, den Lernhabitus der Aufgabenerledigung in unterschiedlichen Ausprägungen zu beschreiben und damit das Konzept des Schülerjobs, wie es Breidenstein (2006) formuliert hat, in Form von Idealtypen (vgl. Weber 1968; Bohnsack 2013) weiter auszudifferenzieren. Die Varianz der Orientierungen an der Aufgabenerledigung steht dabei in Zusammenhang mit der Varianz von Lehrhabitus. Im Folgenden fassen wir vier im empirischen Fallvergleich abstrahierte Typen zusammen, dabei illustrieren wir die idealtypischen Ausformungen des Schülerjobs mit Kurzbeschreibungen von Fällen aus unterschiedlichen Studien. Es handelt sich bei den empirischen Beispielen also nicht um Realtypen, die wir den beschriebenen Typen des Schülerjobs zuordnen, sondern um Beispiele für unterschiedliche Dimensionen des Umgangs der Schüler/innen mit unterrichtlichen Anforderungen, die sich in der empirischen Wirklichkeit des Unterrichts auch überlagern können. ${ }^{4}$

\footnotetext{
4 Zum besseren Verständnis wäre es sicherlich hilfreich, wenn die illustrierenden Fallbeispiele in ausführlichen Fallbeschreibungen präsentiert werden könnten, was hier aus Platzgründen leider nicht für alle vier Typen möglich ist. Wir wählen deshalb Beispiele, die in anderen Publikationen bereits ausführlich dargestellt wurden, damit die Rekonstruktionen ggf. dort nachvollzogen werden können.
} 


\subsubsection{Typ 1: Abliefern eines Arbeitsergebnisses}

Dieser in unseren empirischen Daten häufig auffindbare Typus der Aufgabenerledigung ist dem von Breidenstein skizzierten Schülerjob am nächsten und insbesondere mit der Produktionsorientierung zu vergleichen. Der Typus ist dadurch gekennzeichnet, dass die Schüler/innen im Zusammenhang mit einem Arbeitsauftrag ein mehr oder weniger komplexes Arbeitsergebnis erstellen. Dabei kann es sich um ein Referat, ein Lernplakat, ein ausgefülltes Arbeitsblatt o. ä. handeln. Charakteristisch für den Typus ist, dass hier vor allem Prozesse der formalen Erledigung des Arbeitsauftrags zu beobachten sind. Die Schüler/innen sind in der Regel auf eine effiziente Fertigstellung in der dafür vorgesehenen Zeit orientiert, ohne sich mit dem fachlichen Inhalt der Aufgabe (vertieft) auseinanderzusetzen oder sich mit ihm oder dem Arbeitsergebnis zu identifizieren.

Beispielhaft zeigt sich dieser Typus in der von uns rekonstruierten Sequenz „Fragen formulieren II“ (Asbrand und Martens 2018, S. 274-300). Im fächerübergreifenden Unterricht zum Thema „Sozialstandards in der Textilindustrie“ haben die Schüler/innen die Aufgabe, sich in Gruppen zu unterschiedlichen Unterthemen eigenständig fachliche Expertise zu erarbeiten und sollen Leitfragen für die Themenerschließung formulieren. Die Beispielsequenz fokussiert eine Arbeitsphase einer Schülergruppe, die durch eine Rahmeninkongruenz zwischen den Schülern geprägt ist und in der die Herstellung von Arbeitsfähigkeit (zunächst) mit peerkulturell relevanten Themensetzungen konkurriert. Der Arbeitsauftrag wird im Sinne des Lernhabitus der Schüler rekontextualisiert und auf den Kern des formalen FragenFormulierens reduziert. Dabei schließen sie an den Lehrhabitus eines Arbeitsblattes an, in dem sich das Aufschreiben von Fragen in vorbereitete leere Zeilen als Selbstzweck zeigt, divergent zu der rahmenden Erwartung der Lehrerin an eine eigenständige Konstruktion von Perspektiven zur Aneignung von Fachwissen. Die in das Arbeitsmaterial eingeschriebenen Orientierungsrahmen ermöglichen den Schüler/innen einzelne Aspekte des Lehr-Lernarrangements parzelliert und für sich zu sehen und abzuarbeiten. Die geringe Tiefe der Auseinandersetzung mit dem Unterrichtsgegenstand zeigt sich darin, dass die Schüler schließlich von einer Pinnwand Fragen abschreiben, die bereits am Vortag - zu einer anders gelagerten Aufgabenstellung - formuliert und diskutiert worden waren.

Ein weiteres Beispiel ist die Sequenz „Stationenarbeit“ (Martens 2020), in der Schüler/innen eine Aufgabensammlung zu einer Ganzschrift im Deutschunterricht bearbeiten. Die Aufgabensammlung ist auf eine individuelle Bearbeitung ausgelegt und ist auch als solche bei der Lehrperson abzugeben. Da die Schüler/innen in Tischgruppen sitzen, kommt es während der Bearbeitung zu einem regen Austausch, in dem sich aufgabenbezogene und peerkulturelle Themen überlagern. Die zu bearbeitende Masse an eindimensionalen, eindeutigen und selbsterklärlichen Aufgaben führt zu einer Abarbeitung, die den Charakter der Beiläufigkeit erhält. Während Lückentexte ausgefüllt und vorgedruckte Linien vollgeschrieben werden verbringen die Schüler/innen die Arbeitszeit wesentlich mit sozialen Vergleichen und Distinktionspraktiken in der Peergroup. Fremd- und Selbstpositionierungen nach den Kriterien Schnelligkeit, Selbstständigkeit und Produktivität sind die beherrschenden Themen der Schüler/innen. Auch hier ist zu beobachten, wie der Habitus des Lernmaterials 
die Arbeits- und Sozialdynamik beeinflusst und Anschlussmöglichkeiten an einen Lernhabitus der formalen Aufgabenerledigung und Ablieferung eines Arbeitsergebnisses bietet.

\subsubsection{Typ 2: Mitarbeit in der Unterrichtsinszenierung}

Mit einer Orientierung an der Mitarbeit in der Unterrichtsinszenierung beteiligen sich die Schüler/innen an der Hervorbringung und Stabilisierung des Unterrichts, indem sie mit ihren Lernhabitus an bestimmte lehrerseitige Inszenierungselemente anschließen. Die Mitarbeit an der Unterrichtsinszenierung als Spezifizierung der Orientierung an der Aufgabenerledigung haben wir oben im Interpretationsbeispiel zur Sequenz „Vorbereitung auf die Klassenarbeit“ ausführlich beschrieben: Mit ihren Orientierungen an Erfüllung von Regel- bzw. Zusatzanforderungen stützen die Schüler/innen eine an Differenzierung orientierte Unterrichtsgestaltung der Lehrperson, die letztlich zur Reproduktion und Verfestigung von Leistungspositionierungen der Schüler/innen führt (vgl. Martens 2015). Oft lässt sich eine solche Orientierung an der Mitarbeit in der Unterrichtsinszenierung in der Beteiligung an klassenöffentlichen Unterrichtsgesprächen rekonstruieren, bei denen sich die Schüler/ innen reibungslos in das Frage-Antwort-Evaluations-Schema einpassen (vgl. z.B. die Sequenz „Umgang mit Quellen“ in Asbrand et al. 2018, S. 82 ff.). Sie wirken beim Finden der „,richtigen“ Antworten mit, auch wenn die Fragen der Lehrperson mehrdeutig, uneindeutig oder fachlich problematisch sind und tragen so zur Aufrechterhaltung (der Illusion) einer Sinnhaftigkeit des Unterrichts bei (vgl. z. B. die Sequenz „Evolution hat kein Ziel“ in Martens und Gresch 2018; auch Gresch 2020). Die Orientierung an der Mitarbeit in der Unterrichtsinszenierung haben wir auch in Sequenzen rekonstruiert, in denen Orientierungsrahmen und Orientierungsschemata der Lehrperson Inkonsistenzen aufweisen, wenn also im Unterricht auf der verbalen oder performativen Ebene pädagogische, fachliche oder fachdidaktische Normen aufgerufen werden, die keine Entsprechung in der habituellen Strukturierung der Praxis haben. So zeigt die Sequenz „Morgenkreis im Gymnasium“ (Petersen 2015; Martens et al. 2015a) die Inszenierung eines Erzählkreises zu den Wochenenderlebnissen, die die Lehrperson aber als fragendentwickelndes Unterrichtsgespräch praktiziert. Die Schüler/innen schließen insbesondere an die habituelle Ebene an, indem sie sich auf den unterrichtlichen Charakter des Morgenkreises einstellen und der Lehrperson vor allem schulisch relevante Erlebnisse des Wochenendes (z. B. Erledigung von Hausaufgaben) berichten. Auf fachlicher bzw. fachdidaktischer Ebene zeigt sich die Mitarbeit an der Unterrichtsinszenierung z. B. in einem Geschichtsunterricht, der die Anfertigung einer Quellenkritik für die im Unterricht bearbeiteten historischen Dokumente erwartet, aber keine Anlässe bietet, die Ergebnisse der Kritik für den historischen Erkenntnisprozess relevant zu machen. $\mathrm{Zu}$ beobachten ist die Inszenierung wissenschaftsorientierten Geschichtsunterrichts, der die Schüler/ innen durch eine formalisierte Bearbeitung der Quellenkritikaufgabe entsprechen (vgl. Spieß 2014).

Die Orientierung an der Mitarbeit in der Unterrichtsinszenierung zeigt sich auch in Sequenzen, in denen es um überfachliches Lernen geht. In verschiedenen, von uns interpretierten Sequenzen, reflektieren Schüler/innen ihr Arbeitsverhalten bzw. 
ihre Beteiligung an der Störung des Unterrichts (Martens 2018). In der Interpretation zeigte sich, dass die Reflexionen von den Schüler/innen als schulische Aufgaben, die in das System der schulischen Leistungserbringung und -bewertung integriert sind, wahrgenommen und routiniert erledigt werden. Bezugspunkt ist nicht das eigene Verhalten oder Denken, sondern die Erwartungshaltung der Lehrperson. In der komplementären Interaktion kommt den Schüleräußerungen die Funktion der Gelingensbedingung für die Unterrichtsgestaltung zu, die an Reibungslosigkeit und Effektivität orientiert ist. Durch die lernhabituellen Anschlüsse tragen die Schüler/innen zum Funktionieren des Unterrichts bei, indem sie sich als Reflektierende darstellen, Selbstkritik gegenüber dem gezeigten Arbeits- und Sozialverhalten zum Ausdruck bringen und Selbstoptimierung in Aussicht stellen.

\subsubsection{Typ 3: Wissensreproduktion}

Der Typus der Wissensreproduktion findet sich in Lehr-Lernarrangements, die durch einen instruktivistischen Lehrhabitus bestimmt sind. Die Unterrichtsinteraktion ist dabei formal durch die Lehrperson und/oder die Unterrichtsmaterialien gesteuert und auch die Inhalte sind als eindeutiges Wissen weitgehend vorgegeben. Die Anforderung an die Schüler/innen besteht darin, das ,richtige“ Wissen benennen zu können. Im Modus der Aufgabenerledigung kommen die Schüler/innen dieser Anforderung nach und reproduzieren die als relevant ausgewiesenen fachlichen Sachverhalte. Eine darüberhinausgehende Auseinandersetzung mit den Unterrichtsinhalten kann in Unterrichtssituationen, die durch den Modus der Wissensreproduktion bestimmt sind, nicht beobachtet werden.

Ein Beispiel für die Relationierung von instruktivistischem Lehrhabitus und den Lernhabitus der Wissensreproduktion ist eine Sequenz aus dem Mathematikunterricht (vgl. Asbrand et al. 2020): Die Lehrerin zeigt geometrische Körper, die als Anschauungsobjekte fungieren, und erwartet von den Schüler/innen, dass diese mit dem korrekten Fachbegriff (Quader) bezeichnet und die Merkmale des jeweiligen Körpers genannt werden können. Diese Abfrage mathematischen Wissens, über das die Schüler/innen bereits verfügen, bezeichnet die Lehrerin als Training; mathematisches Fachwissen wird also als klar definiertes Wissen verstanden, das durch Wiederholung und Einübung ,antrainiert“ werden kann.

Auch in der bereits erwähnten Studie zu Themen des Lernbereichs Globale Entwicklung (Wettstädt und Asbrand 2013, 2014; Kater-Wettstädt 2015), finden sich Beispiele für die Relationierung von instruktivistischem Lehrhabitus und einem Lernhabitus der Wissensreproduktion. Der rekonstruierte Lehrhabitus, den wir als themenvermittelnd bezeichnet haben, zeichnet sich dadurch aus, dass ethische Fragestellungen nicht diskursiv und multiperspektivisch bearbeitet werden, sondern zu komplexen Themen ganz bestimmte moralische Positionen vertreten werden (Wettstädt und Asbrand 2014) oder verschiedene Perspektiven eines Sachverhalts als eindeutige Information thematisiert werden (Wettstädt und Asbrand 2013). Hierzu passt eine Lernpraxis, moralisch aufgeladene Handlungsaufforderungen (z. B. fair gehandelte Textilien zu kaufen) bzw. standortgebundene Positionen (z. B. die Verbraucher/innenperspektive) als schulisch relevantes Wissen zu reproduzieren, ohne 
dass es zu einer tieferen Auseinandersetzung mit den kontroversen Inhalten oder einer Reflexion über die Perspektivität von Wissen käme.

Schließlich finden sich auch in der Studie zum Umgang mit Quellen im Geschichtsunterricht (Spieß 2014) mehrere Beispiele für das Passungsverhältnis von einem an Wissensvermittlung orientierten Lehrhabitus und einem Lernhabitus der Wissensreproduktion. In Unterrichtssequenzen, in denen mit Textquellen gearbeitet wurde, werden diese durch die Lehrperson als ein Text gerahmt, dem Informationen über ein historisches Ereignis entnommen werden können. Quelle und historischer Sachverhalt werden gleichgesetzt. Im Modus der Aufgabenerledigung kommen die Schüler/innen der Aufforderung nach, dem Text Informationen zu entnehmen und diese im Unterrichtsgespräch oder in einem schriftlichen Arbeitsprodukt wiederzugeben.

\subsubsection{Typ 4: Fachliche Eigenkonstruktionen der Schüler/innen}

In den Studien aus unserem Arbeitsbereich zeigt sich, dass auch die - aus einer normativen Perspektive wünschenswerte - eigenständige Aneignung von Fachwissen durch Schüler/innen im Modus der Aufgabenerledigung erfolgt. Denn auch fachliche Eigenkonstruktionen konnten als Reaktionsweisen auf eine heteronome Anforderungsstruktur rekonstruiert werden. Einen Lernhabitus der Ko-Konstruktion von fachlichem Wissen identifizieren wir, wenn auf Seiten des Lehr-Lernarrangements zwei Aspekte zusammenkommen: 1. Die Aufgabenstellungen sind eindeutig fachlich fokussiert, nehmen aber in Bezug auf den konkreten Unterrichtsgegenstand keine inhaltlichen Setzungen vor; Aufgaben in diesem Sinne sind ein fachlich definierter und strukturierter Rahmen für die Eigenkonstruktionen der Lernenden, ohne diese zu determinieren; 2. Der Lehrhabitus der Lehrperson ist durch Zutrauen in die Fähigkeit der Schüler/innen, sich selbständig Fachwissen aneignen zu können, bestimmt.

Beispiele für diese Relationierung eines als konstruktivistisch zu bezeichnenden Lehrhabitus mit dem Lernhabitus der Wissensaneignung finden sich in der Studie zum Lernbereich Globale Entwicklung (Kater-Wettstädt 2015), vor allem im Rahmen der videografierten Unterrichtseinheit zu Sozialstandards in der Textilproduktion (vgl. auch Martens und Asbrand 2017). Auch in Sequenzen aus einer Unterrichtseinheit zu Globalisierung im 10. Jahrgang einer Gesamtschule zeigt sich, wie die diskursive Unterrichtsgestaltung durch den Lehrer und die Methode der Zukunftswerkstatt, deren Arbeitsschritte von den Schüler/innen abgearbeitet bzw. „erledigt“" werden, zugleich eine inhaltliche Offenheit schaffen, die den Schüler/ innen die Entwicklung eigener Zukunftsszenarien zu Fragen der Globalisierung und Reflexionen über deren Realisierung ermöglichen (Wettstädt und Asbrand 2014).

Auch in einer Studie zum Mathematikunterricht ließ sich das beschriebene Passungsverhältnis rekonstruieren. Aufgabenerledigung lässt sich hier schüler/ innenseitig als eine Ko-Konstruktion der mathematischen Modellierung von realweltlichen Problemen im Modus triftiger Argumentationen und unter Anwendung von Vorwissen beobachten. Der rekonstruierte Lehrhabitus zeichnet sich auch hier durch das Zutrauen in die Fähigkeiten der Schüler/innen aus, die Aufgabenstellung ist durch fachliche Strukturierung und inhaltliche Offenheit bestimmt: zu einem 
konkreten Problem sollen Modellierungen und Lösungswege entwickelt werden (Schreyer et al. eingereicht).

Schließlich haben wir im Rahmen der Studie zum Umgang mit Quellen im Geschichtsunterricht eine Unterrichtseinheit videografiert und analysiert, in der eine Sachquelle Ausgangspunkt der Auseinandersetzung mit den fachlichen Inhalten ist (Martens et al. 2015b; Asbrand und Martens 2020). Die Beschäftigung mit dem historischen Artefakt erfolgt im Rahmen einer unterrichtlichen Aufgabenstellung und im Modus der Aufgabenerledigung. Gleichzeitig geht mit der Authentizität und der Materialität der Quelle einher, dass ihr ,Informationsgehalt“ ungewiss ist und rekonstruiert werden muss. Auch nimmt die Aufgabenstellung, in der sich der Lehrhabitus als Orientierung an Eigenkonstruktionen der Schüler/innen dokumentiert, keine inhaltlichen Setzungen vor und fordert die Schüler/innen zum Beobachten und FragenStellen auf. Die videografierten Schüler/innen lassen sich im Modus der Erledigung auf die Aufgabe ein, untersuchen den Gegenstand, „tasten“ sich an den historischen Kontext der Quelle heran, suchen gemeinsam nach Erkenntnissen und akzeptieren, dass Fragen offen und ihr Wissen über den historischen Kontext aspekthaft bleiben.

\section{Schülerjob revisited: Diskussion und Ausblick}

Wir haben uns in diesem Beitrag der Fragestellung Breidensteins, ,wie Jugendliche mit schulischem Unterricht umgehen, wie sie die situativen und interaktiven Anforderungen des Unterrichts handhaben“ (2006, S. 5) angeschlossen und haben einen Vorschlag zur Ausdifferenzierung des Schülerjobs entlang typischer Ausformungen der für die Teilnahme an Unterricht grundlegenden Orientierung an der Aufgabenerledigung vorgestellt. Die vier Typen sind das Ergebnis der theoretischen Abstraktion vorliegender Untersuchungsbefunde aus mehreren Studien der fachdidaktischen und erziehungswissenschaftlichen Unterrichtsforschung.

Eine Besonderheit von Unterricht ist, dass in der Interaktion zwischen Lehrperson und Schüler/innen (häufig) differente Orientierungsrahmen aufeinandertreffen. In Fragestellungen, die auf das Verhältnis von fachlichem Lehren und Lernen bzw. auf den Umgang mit schulischen Erwartungen und den schulischen Wissenserwerb fokussiert sind, können diese Differenzen als Passungsverhältnisse rekonstruiert werden. Das Konzept der Passungsverhältnisse kann also erklären, warum Unterricht häufig reibungslos verläuft, obwohl die Akteur/innen mit unterschiedlichen habituellen Orientierungen am Unterricht teilnehmen (vgl. Martens und Asbrand 2017). Empirisch zeigt sich, dass Lehr- und Lernhabitus (im Sinne eines Mehrebenenmodells) im Unterricht füreinander Kontexte darstellen. In den von uns herangezogenen Studien konnten typische Muster der Relationierung von bestimmten im Unterricht jeweils wirksamen Lehrhabitus und den Lernhabitus der Schüler/innen rekonstruiert werden, die wir im Rahmen einer relationalen Typenbildung (Nohl 2013a) aufeinander bezogen und in diesem Beitrag systematisiert haben. Für die Unterrichtsforschung bietet sich die relationale Typenbildung an, da sie das In-Beziehung-Setzen von in der dokumentarischen Interpretation der Unterrichtsvideografien rekonstruierten Merkmalen der Unterrichtsinteraktion mit ebenfalls empirisch rekonstruierten 
Orientierungsrahmen und Lernpraxen von Schüler/innen erlaubt (vgl. Asbrand und Martens 2018, S. 40ff.).

Die Differenzierung des Schülerjobs auf der Basis der wissenssoziologisch fundierten Dokumentarischen Methode stellt zu Breidensteins Rekonstruktion des im Unterricht wirksamen komplexen sozialen Gefüges aus Peer- und Unterrichtskultur und der Praktiken ihrer Herstellung eine fruchtbare Ergänzung dar. Die Differenzierungen sind auf mehreren Ebenen angesiedelt und betreffen die Fachlichkeit des Unterrichts, die Relationierung zu anderen Habitusdimensionen, die Relationierung zu den Lehrhabitus sowie die Frage des Geltungsbereichs des „Schülerjob“-Theorems:

Fachlichkeit des Unterrichts: In ihrer Fokussierung auf kommunikative und konjunktive Wissensbestände ermöglicht die dokumentarische Unterrichtsforschung einen empirischen Zugang zu fachlichen Wissensbeständen und fachspezifischen Routinen und kann somit Fachlichkeit als unterrichtliche Dimension in besonderer Weise in den Blick nehmen.

Relationierung zu anderen Habitusdimensionen: Der methodische Zugang über die Dokumentarische Methode erlaubt weitere Differenzierungen in der Betrachtung des Schülerjobs: Die komparative Analyse mehrdimensionaler Erfahrungsräume ermöglicht es, Orientierungsrahmen, die in peerkulturellen Erfahrungsräumen entstehen und sich vor allem in der Peerinteraktion aktualisierten und Orientierungsrahmen, die auf das Lernen und die Bewältigung von unterrichtlichen Anforderungen bezogen sind, voneinander analytisch zu unterscheiden. Im Sinne der Dokumentarischen Methode, die bestrebt ist, die mehrdimensionale Überlagerung von konjunktiven Erfahrungen zu rekonstruieren, stellt der Lernhabitus mit der für ihn charakteristischen Orientierung an der Aufgabenerledigung eine Dimension des mehrdimensional verfassten Schüler/innenhabitus dar. Der Vorteil dieser Analyseeinstellung besteht darin, dass einzelne Dimensionen analytisch unterschieden werden können und damit deren jeweilige Bedeutung in der sozialen Erfahrungsaufschichtung sowie die Bedeutung füreinander in der sozialen Alltagspraxis rekonstruiert werden kann.

Relationierung zu den Lehrhabitus: Breidenstein (2006) macht deutlich, dass durch seine Forschung zum Schülerjob das „Bild vom Unterricht als einer die ganze Klasse gemeinsam und gleichermaßen betreffende Veranstaltung, deren Sinn in Lehr-Lernprozessen liegt, [...] fragwürdig [wird]. Die Vielfalt, die Ungleichzeitigkeit und das Fragmentarische des Geschehens werden evident, und die Frage, was eigentlich den ,Unterricht" ausmacht, wird immer komplizierter.“ (ebd., S. 10). An dieses Ergebnis schließen wir an und zeigen in der Rekonstruktion von Passungsverhältnissen von Lehr- und Lernhabitus deren Abhängigkeit voneinander als wechselseitige Kontexte im Sinne einer Bedingung der Möglichkeit. Differenz der Teilnahmemöglichkeiten an Unterricht zeigt sich empirisch an den unterschiedlichen Lernhabitus und den damit verbundenen Passungsverhältnissen, die in einer Lerngruppe ko-existieren. Diese stellen sich empirisch als nicht einheitlich heraus - auch das durch die 
Lehrperson gestaltete „Unterrichtsangebot“ ist nicht zwangsläufig mit sich selbst konsistent oder kongruent.

Geltungsbereich des ,Schülerjob“-Theorems: Auch hinsichtlich des Geltungsbereichs erweitern unsere Analysen die Befunde Breidensteins. Er fokussiert in seiner Studie zum Schülerjob auf Unterricht im 7. und 8. Schuljahr und hebt hervor, dass diese Phase der Hochadoleszenz durch eine spezifische „Schulferne“ geprägt sein mag. Zugleich stellt er die Vermutung auf, dass sich insbesondere ,in diesem Alter ein instrumentell-strategischer Umgang mit der Schule herauszubilden [scheint], der sich in dem Bild des ,Schülerjobs" verdichtet" (Breidenstein 2006, S. 11). Auf der Basis unseres umfangreichen empirischen Materials aus allen Schulstufen können wir festhalten, dass die Basistypik der Orientierung an Aufgabenerledigung bei Grundschüler/innen (Petersen 2015; Hackbarth 2017) ebenso zu finden ist wie bei Oberstufenschüler/innen (Schuster in Vorb.). Zu fragen ist, ob der von Breidenstein konstatierte starke Einschlag instrumentell-strategischer Umgangsweisen, die auch mit opportunistisch-distanzierenden Praktiken einhergehen, charakteristisch für die Hochphase der Adoleszenz sind. Für die Entwicklung von Aufgabenerledigungsorientierungen über die Schulzeit hinweg fehlen allerdings längsschnittliche Untersuchungen, die über den von Petersen (2015) untersuchten Übergang zwischen Primarund Sekundarstufe hinausgehen. Allerdings zeigt diese Studie, dass der durch die Orientierung an Aufgabenerledigung bestimmte Lernhabitus bereits in der Grundschule erworben wird und sich von den ersten Schulwochen an in Passung zu entsprechenden Lehrhabitus der weiterführenden Schulen befindet (vgl. auch Petersen, Asbrand und Martens 2020).

Funding Open Access funding enabled and organized by Projekt DEAL.

Open Access Dieser Artikel wird unter der Creative Commons Namensnennung 4.0 International Lizenz veröffentlicht, welche die Nutzung, Vervielfältigung, Bearbeitung, Verbreitung und Wiedergabe in jeglichem Medium und Format erlaubt, sofern Sie den/die ursprünglichen Autor(en) und die Quelle ordnungsgemäß nennen, einen Link zur Creative Commons Lizenz beifügen und angeben, ob Änderungen vorgenommen wurden.

Die in diesem Artikel enthaltenen Bilder und sonstiges Drittmaterial unterliegen ebenfalls der genannten Creative Commons Lizenz, sofern sich aus der Abbildungslegende nichts anderes ergibt. Sofern das betreffende Material nicht unter der genannten Creative Commons Lizenz steht und die betreffende Handlung nicht nach gesetzlichen Vorschriften erlaubt ist, ist für die oben aufgeführten Weiterverwendungen des Materials die Einwilligung des jeweiligen Rechteinhabers einzuholen.

Weitere Details zur Lizenz entnehmen Sie bitte der Lizenzinformation auf http://creativecommons.org/ licenses/by/4.0/deed.de.

\section{Literatur}

Asbrand, B., \& Martens, M. (2018). Dokumentarische Unterrichtsforschung. Wiesbaden: Springer VS. Asbrand, B., \& Martens, M. (2020). Rekonstruktion von Lernprozessen im Unterricht - Herausforderungen und Vorschläge aus der Perspektive der dokumentarischen Unterrichtsforschung. In M. Corsten, K. Hauenschild, M. Pierburg, B. Schmidt-Thieme, U. Schütte, S. Zourelidis \& D. Wolff (Hrsg.), Qualitative Videoanalyse in Schule und Unterricht (S. 112-125). Weinheim, Basel: Juventa Beltz. 
Asbrand, B., Martens, M., \& Schuster, M. (2018). Unterrichtsforschung in kulturwissenschaftlichen Fächern: Herausforderungen und Perspektiven. In M. Schambeck \& U. Riegel (Hrsg.), Was im Religionsunterricht so läuft - Wege und Ergebnisse religionspädagogischer Unterrichtsforschung (S. 74-90). Freiburg: Herder.

Asbrand, B., Martens, M., \& Nohl, A.-M. (2020). Pädagogische Interaktionen in der dokumentarischen Interpretation von Videografien. JDM - Jahrbuch Dokumentarische Methode, 2-3, 299-328.

Bohnsack, R. (2013). Typenbildung, Generalisierung und komparative Analyse: Grundprinzipien der dokumentarischen Methode. In R. Bohnsack, I. Nentwig-Gesemann \& A.-M. Nohl (Hrsg.), Die dokumentarische Methode und ihre Forschungspraxis. Grundlagen qualitativer Sozialforschung (S. 241-270). Wiesbaden: Springer VS.

Bohnsack, R. (2014). Rekonstruktive Sozialforschung. Einfuihrung in qualitative Methoden. Opladen: Budrich.

Bourdieu, P. (1982). Die feinen Unterschiede. Kritik der gesellschaftlichen Urteilskraft. Frankfurt/Main: Suhrkamp.

Bourdieu, P., \& Passeron, J. (1971). Die Illusion der Chancengleichheit. Untersuchungen zur Soziologie des Bildungswesens am Beispiel Frankreichs. Stuttgart: Klett.

Breidenstein, G. (2006). Teilnahme am Unterricht. Ethnographische Studien zum Schülerjob. Wiesbaden: VS.

Breidenstein, G. (2008). Schulunterricht als Gegenstand ethnographischer Forschung. In B. Hühnersdorf, C. Maeder \& B. Müller (Hrsg.), Ethnographie und Erziehungswissenschaft. Methodologische Reflexionen und empirische Annäherungen (S. 107-117). Weinheim: München.

Breidenstein, G., \& Menzel, C. (2014). „Arbeitszeit ist zum Arbeiten da!“ - Zur Arbeits-Terminologie im individualisierten Unterricht. Zeitschrift für Grundschulforschung, 7(2), 179-193.

Breidenstein, G., Dorow, S., Menzel, C., \& Rademacher, S. (2012). Anstellen statt Melden - Die Warteschlange im individualisierten Unterricht. In F. Hellmich, S. Förster \& F. Hoya (Hrsg.), Bedingungen des Lehrens und Lernens in der Grundschule (S. 77-80). Wiesbaden: Springer VS.

Bremer, H., \& Lange-Vester, A. (2014). Die Pluralität der Habitus- und Milieuformen bei Lernenden und Lehrenden. Theoretische und methodologische Überlegungen zum Verhältnis von Habitus und sozialem Raum. In W. Helsper, R.-T. Kramer \& S. Thiersch (Hrsg.), Schülerhabitus. Theoretische und empirischer Analysen zum Bourdieuschen Theorem der kulturellen Passung (S. 56-81). Wiesbaden: VS.

Gibson, A., \& Helsper, W. (2018). Passungstypologie von individuellen und institutionellen Schülerhabitus. Eine mehrebenenanalytische, relationale Typenbildung. In R. Bohnsack, N.F. Hoffmann \& I. Nentwig-Gesemann (Hrsg.), Typenbildung und Dokumentarische Methode. Forschungspraxis und methodologische Grundlagen (S. 151-169). Opladen: Barbara Budrich.

Goffman, I. (1971). Verhalten in sozialen Situationen. Strukturen und Regeln der Interaktion im öffentlichen Raum. Gütersloh: Bertelsmann.

Gresch, H. (2020). Teleological explanations in evolution classes: video-based analyses of teaching and learning processes across a seventh-grade teaching unit. Evolution: Education and Outreach, 13(10), $1-19$.

Gresch, H., \& Martens, M. (2019). Teleology as a tacit dimension of teaching and learning evolution: a sociological approach to classroom interaction in science education. Journal for Research in Science Teaching, 56(2), 243-269.

Hackbarth, A. (2017). Inklusionen und Exklusionen in aufgabenbezogenen Schülerinteraktionen. Bad Heilbrunn: Klinkhardt.

Heinze, T. (1980). Schülertaktiken. München: Urban und Schwarzenberg.

Helmke, A. (2017). Unterrichtsqualität und Lehrerprofessionalität. Diagnose, Evaluation und Verbesserung des Unterrichts (7. Aufl.). Seelze-Velber: Klett.

Helsper, W. (2001). Praxis und Reflexion. Die Notwendigkeit einer „doppelten Professionalisierung“ des Lehrers. Journal für LehrerInnenbildung, 1(3), 7-15.

Helsper, W. (2018). Lehrerhabitus. Lehrer zwischen Herkunft, Milieu und Profession. In A. Paseka, M. Keller-Schneider \& A. Combe (Hrsg.), Ungewissheit als Herausforderung für pädagogisches Handeln (S. 105-140). Wiesbaden: Springer VS.

Helsper, W., Gibson, A., Kilias, W., Kotzyba, K., \& Niemann, M. (2020). Veränderungen im Schülerhabitus? Die Schülerschaft exklusiver Gymnasien von der 8. Klasse bis zum Abitur. Wiesbaden: Springer VS.

Helsper, W., Kramer, R.-T., \& Thiersch, S. (Hrsg.). (2014). Schülerhabitus. Theoretische und empirische Analysen zum Bourdieuschen Theorem der kulturellen Passung. Wiesbaden: Springer VS. 
Jackson, P. W. (1973). Die Welt des Schülers. In W. Edelstein \& D. Hopf (Hrsg.), Bedingungen des Bildungsprozesses. Psychologische und pädagogische Forschungen zum Lehren und Lernen in der Schule (S. 13-27). Stuttgart: Klett.

Jackson, P.W. (1975). Einübung in eine bürokratische Gesellschaft: Zur Funktion der sozialen Verkehrsformen im Klassenzimmer. In J. Zinnecker (Hrsg.), Der Heimliche Lehrplan. Untersuchungen zum Schulunterricht (S. 19-34). Weinheim: Beltz.

Kater-Wettstädt, L. (2015). Unterricht im Lernbereich Globale Entwicklung - der Kompetenzerwerb und seine Bedingungen. Münster: Waxmann.

Kramer, R.-T. (2014). Kulturelle Passung und Schülerhabitus - Zur Bedeutung der Schule für Transfonnationsprozesse des Habitus. In W. Helsper, R.-T. Kramer \& S. Thiersch (Hrsg.), Schülerhabitus. Theoretische und empirische Analysen zum Bourdieuschen Theorem der kulturellen Passung (S. 183-205). Wiesbaden: Springer VS.

Kramer, R.-T. (2015). Kulturelle Passung und sanfte Eliminierung. Heterogenität und Homogenisierung in der Perspektive von Pierre Bourdieu. In J. Budde, N. Blasse, A. Bossen \& G. Rißler (Hrsg.), Heterogenitätsforschung. Empirische und theoretische Perspektiven (S. 34-56). Weinheim: Beltz Juventa.

Kramer, R.-T., \& Pallesen, H. (Hrsg.). (2019). Lehrerhabitus. Theoretische und empirische Beiträge zu einer Praxeologie des Lehrerberufs. Bad Heilbrunn: Klinkhardt.

Kreft, A. (2020). Transkulturelle Kompetenz und literaturbasierter Fremdsprachenunterricht. Eine rekonstruktive Studie zum Einsatz von fictions of migration im Fach Englisch. Berlin: Peter Lang.

Luhmann, N. (2002). Das Erziehungssystem der Gesellschaft. Frankfurt am Main: Suhrkamp.

Mannheim, K. (1980). Strukturen des Denkens. Frankfurt am Main: Suhrkamp.

Martens, M. (2015). Differenz und Passung: Differenzkonstruktionen im individualisierenden Unterricht der Sekundarstufe. Zeitschrift für Qualitative Forschung, 16(2), 211-230.

Martens, M. (2018). Reflektieren als unterrichtliche Aufgabe: Zur Passung von Lehr- und Lernkompetenzen im individualisierten Unterricht. In K. Rabenstein, K. Kunze, M. Martens, T.-S. Idel, M. Proske \& S. Strauß (Hrsg.), Individualisierung von Unterricht: Transformationen - Wirkungen - Reflexionen (S. 88-102). Bad Heilbrunn: Klinkhardt.

Martens, M. (2020). Heterogenität aus Sicht von Schüler*innen: Empirische Befunde zum individualisierten Unterricht in der Sekundarstufe. In M. Kampshoff \& C. Wiepcke (Hrsg.), Vielfalt in Schule und Unterricht. Konzepte und Debatten im Zeichen der Heterogenität (S. 125-137). Stuttgart: Kohlhammer.

Martens, M., \& Asbrand, B. (2017). Passungsverhältnisse: Methodologische und theoretische Reflexionen zur Interaktionsorganisation des Unterrichts. Zeitschrift für Pädagogik, 63(1), 72-90.

Martens, M., \& Gresch, H. (2018). Ambivalente Fachlichkeiten: Die (Re)Produktion fachlicher Vorstellungen im/durch Biologieunterricht. In M. Martens, K. Rabenstein, K. Bräu, M. Fetzer, H. Gresch, I. Hardy \& C. Schelle (Hrsg.), Konstruktionen von Fachlichkeit: Ansätze, Erträge und Diskussionen in der empirischen Unterrichtsforschung (S. 275-288). Bad Heilbrunn: Klinkhardt.

Martens, M., Asbrand, B., \& Spieß, C. (2015b). Lernen mit Dingen - Prozesse zirkulierender Referenz im Unterricht. Zeitschrift für interpretative Schul- und Unterrichtsforschung (ZISU), 4, 48-63.

Martens, M., Petersen, D., \& Asbrand, B. (2015a). Die Materialität von Lernkultur. Methodische Überlegungen zur dokumentarischen Analyse von Unterrichtsvideografien. In R. Bohnsack, B. Fritzsche \& M. Wagner-Willi (Hrsg.), Dokumentarische Film und Videointerpretation. Methodologie und Forschungspraxis (S. 179-203). Opladen: Budrich.

Nohl, A.-M. (2013a). Relationale Typenbildung und Mehrebenenvergleich. Neue Wege der dokumentarischen Methode. Wiesbaden: Springer VS.

Nohl, A.-M. (2013b). Komparative Analyse: Forschungspraxis und Methodologie dokumentarischer Interpretation. In R. Bohnsack, I. Nentwig-Gesemann \& A.-M. Nohl (Hrsg.), Die dokumentarische Methode und ihre Forschungspraxis. Grundlagen qualitativer Sozialforschung (S. 271-293). Wiesbaden: Springer VS.

Petersen, D. (2015). Anpassungsleistungen und Konstruktionsprozesse beim Grundschulübergang. Wiesbaden: Springer VS.

Petersen, D., \& Asbrand, B. (2013). Anpassungsleistungen von Schülerinnen und Schülern beim Übergang von der Grundschule in die weiterführenden Schulen. Zeitschrift für Qualitative Forschung, 14(1), 49-65.

Petersen, D., Asbrand, A., \& Martens, M. (2020). Der Übergang von der Grundschule in die Sekundarstufe: Zur Rekonstruktion unterrichtlicher Passungsverhältnisse im qualitativen Längsschnitt. In S. Thiersch (Hrsg.), Qualitative Längsschnittforschung: Bestimmungen, Forschungspraxis und Reflexionen (S. 273-294). Opladen: Budrich. 
von Rosenberg, F. (2014). Schülerhabitus und Habitustransformation. Peergroups als Potentiale für Bildungsprozesse. In W. Helsper, R.-T. Kramer \& S. Thiersch (Hrsg.), Schülerhabitus. Theoretische und empirische Analysen zum Bourdieuschen Theorem der kulturellen Passung (S. 274-290). Wiesbaden: Springer VS.

Schreyer, P., Martens, M., \& Klieme, E. Kognitive Aktivierung in der Lehrer-Schüler-Interaktion. Perspektiven der Dokumentarischen Unterrichtsforschung. In A.-K. Praetorius, M. Brinkmann, W. Rogh, \& M. Weber-Spanknagel (Hrsg.), Triangulation von videobasierter quantitativer und qualitativer Unterrichtsforschung. Wiesbaden: Springer VS. eingereicht.

Schuster, M. Praktiken des Umgangs mit Fachtexten in gesellschaftswissenschaftlichen Fächern in der Sekundarstufe II. Dissertation in Vorbereitung. Frankfurt am Main: Goethe-Universität Frankfurt am Main.

Spieß, C. (2014). Quellenarbeit im Geschichtsunterricht. Die empirische Rekonstruktion von Kompetenzerwerb im Umgang mit Quellen. Göttingen: V+R unipress.

Thiersch, S. (2014). Schülerhabitus und familialer Bildungshabitus - Zur Genese von Bildungskarrieren und -entscheidungen. In W. Helsper, R.-T. Kramer \& S. Thiersch (Hrsg.), Schülerhabitus. Theoretische und empirische Analysen zum Bourdieuschen Theorem der kulturellen Passung (S. 205-225). Wiesbaden: Springer VS.

Weber, M. (1968). Gesammelte Aufsätze zur Wissenschaftslehre. Tübingen: Mohr.

Wettstädt, L., \& Asbrand, B. (2013). Unterricht im Lernbereich Globale Entwicklung: Perspektivität als Herausforderung. In U. Riegel \& K. Macha (Hrsg.), Videobasierte Kompetenzforschung in den Fachdidaktiken (S. 183-197). Münster: Waxmann.

Wettstädt, L., \& Asbrand, B. (2014). Handeln in der Weltgesellschaft. Zum Umgang mit Handlungsaufforderungen im Unterricht zu Themen des Lernbereichs Globale Entwicklung. Zeitschrift für internationale Bildungsforschung und Entwicklungspädagogik, 36(1), 4-12.

Zinnecker, J. (1978). Die Schule als Hinterbühne oder Nachrichten aus dem Unterlebender Schüler. In J. Zinnecker \& G.-B. Reinert (Hrsg.), Schüler im Schulbetrieb. Berichte und Bilder vom Lernalltag, von Lernpausen und vom Lernen in den Pausen (S. 29-121). Reinbek bei Hamburg: Rohwohlt. 